\title{
ACCELERATION OF COCKTAIL IONS AT THE JAERI AVF CYCLOTRON
}

\author{
M. Fukuda, K. Arakawa, S. Okumura, T. Nara, I. Ishibori, Y. Nakamura, W. Yokota, \\ T. Agematsu, H. Tamura, JAERI, Takasaki, Gunma, Japan
}

\begin{abstract}
Ion species and energy of a cyclotron beam can be quickly changed using a cocktail acceleration technique. Cocktail beams of mass-to-charge (M/Q) ratio of 4 and 5 have been developed at the JAERI AVF cyclotron. Purity of the beam depends on the M/Q resolution of the cyclotron. The resolution has been estimated theoretically and experimentally. A radial spread of an internal beam bunch caused by phase slip of particles with a different $\mathrm{M} / \mathrm{Q}$ value has been observed using a pulsed incident beam. For practical operation, some methods have been devised for avoiding intermixture of different ion species in a beam extracted from the cyclotron.
\end{abstract}

\section{INTRODUCTION}

Various ion species with a wide range of energy is available at the JAERI AVF cyclotron facilities [1] for research on materials science and biotechnology. Beam time required for the research ranges from several hours to a few days, relatively shorter than the use for nuclear physics. Frequent change of ion species and/or energy decreases the rate of cyclotron operation, because one through two hours are needed for changing and optimizing all the parameters of the whole cyclotron system. Fast change of the beam without loss of time is required for efficient use of the beam time.

The cocktail acceleration technique [2] is an optimum method for changing ion species and energy quickly. Different ion species having almost identical M/Q values produced by an ECR ion source are simultaneously injected into the cyclotron. The cyclotron parameters are optimized for one of the cocktail ions. The ion is fully accelerated under the isochronous condition given by

$$
2 \pi \frac{f_{R F}}{h}=\frac{Q}{M} \frac{B_{0}}{m_{0} c^{2}},
$$

where $f_{R F}$ is an RF frequency, $h$ a harmonic number, $B_{0}$ magnetic field for isochronism, $\mathrm{m}_{0}$ unified atomic mass unit. Other ions with different $\mathrm{M} / \mathrm{Q}$ values are gradually shifted an additional amount in phase, given by

$$
\Delta \sin \phi=2 \pi h N \frac{\Delta(M / Q)}{M / Q}
$$

where $\mathrm{N}$ is a turn number, and $\Delta \sin \phi$ is expressed as $\left(\sin \phi-\sin \phi_{0}\right)$, that is the change in phase from beginning of acceleration. The ions are phased out when the change in phase becomes +1 or -1 . Other ion species can be extracted from the cyclotron by slightly changing the frequency by $\Delta \mathrm{f}_{\mathrm{RF}} / \mathrm{f}_{\mathrm{RF}}$ or the magnetic field by $\Delta \mathrm{B} / \mathrm{B}$ which is proportional to $\Delta(\mathrm{M} / \mathrm{Q}) /(\mathrm{M} / \mathrm{Q})$. Changing of the frequency or the magnetic field is completed within a few minutes.

In addition the energy is approximately proportional to the mass of ions, which offers different linear energy transfer (LET) in a target material. Thus the cocktail beam has great merit in saving the beam time and in covering the wide range of LET.

\section{$2 \mathrm{M} / \mathrm{Q}$ RESOLUTION}

In the development of a cocktail beam using ${ }^{12} \mathrm{C}^{3+},{ }^{16} \mathrm{O}^{4+}$ and ${ }^{20} \mathrm{Ne}^{5+}$ ions, the ${ }^{12} \mathrm{C}^{3+}$ and ${ }^{20} \mathrm{Ne}^{5+}$ ions were observed in the beam optimized for the ${ }^{16} \mathrm{O}^{4+}$ ion [3]. A series of the $\mathrm{M} / \mathrm{Q}=4$ cocktail ions available at the JAERI AVF cyclotron are listed in Table 1 together with the M/Q difference from the nearest M/Q values. The M/Q difference of ${ }^{12} \mathrm{C}^{3+}$ and ${ }^{20} \mathrm{Ne}^{5+}$ ions from the ${ }^{16} \mathrm{O}^{4+}$ ion is $3.2 \times 10^{-4}$ and $0.6 \times 10^{-4}$, respectively. The M/Q resolution of the cyclotron was estimated to investigate the intermixture of the different ion species.

Table 1: Parameters of the $\mathrm{M} / \mathrm{Q}=4$ cocktail ions

\begin{tabular}{|c|cc|c|c|}
\hline Ion & $\mathrm{M} / \mathrm{Q}$ & $\Delta(\mathrm{M} / \mathrm{Q}) /(\mathrm{M} / \mathrm{Q})$ & $\mathrm{E}(\mathrm{MeV})$ & $\mathrm{f}_{\mathrm{RF}}(\mathrm{MHz})$ \\
\hline${ }^{4} \mathrm{He}^{+}$ & 4.00210 & $6.6 \times 10^{-4}$ & 25 & 11.9073 \\
${ }^{12} \mathrm{C}^{3+}$ & 3.99947 & $3.2 \times 10^{-4}$ & 75 & 11.9152 \\
${ }^{16} \mathrm{O}^{4+}$ & 3.99818 & $0.6 \times 10^{-4}$ & 100 & 11.9190 \\
${ }^{20} \mathrm{Ne}^{5+}$ & 3.99794 & $5.6 \times 10^{-4}$ & 125 & 11.9197 \\
${ }^{40} \mathrm{Ar}^{10+}$ & 3.99569 & $1.1 \times 10^{-4}$ & 250 & 11.9264 \\
${ }^{84} \mathrm{Kr}^{21+}$ & 3.99524 & & 525 & 11.9278 \\
\hline
\end{tabular}

\subsection{Theoretical Estimation}

The $\mathrm{M} / \mathrm{Q}$ resolution defined by $\mathrm{R}=|(\mathrm{M} / \mathrm{Q}) / \Delta(\mathrm{M} / \mathrm{Q})|$ is equivalent to $\left|f_{R F} / \Delta f_{R F}\right|$, which is given by differentiating the Eq. (1). The resolution can be estimated from a frequency range at which particles can reach to an extraction radius. Change in phase for the frequency shift $\Delta \mathrm{f}_{\mathrm{RF}} / \mathrm{f}_{\mathrm{RF}}$ with non-relativistic approximation is given by 


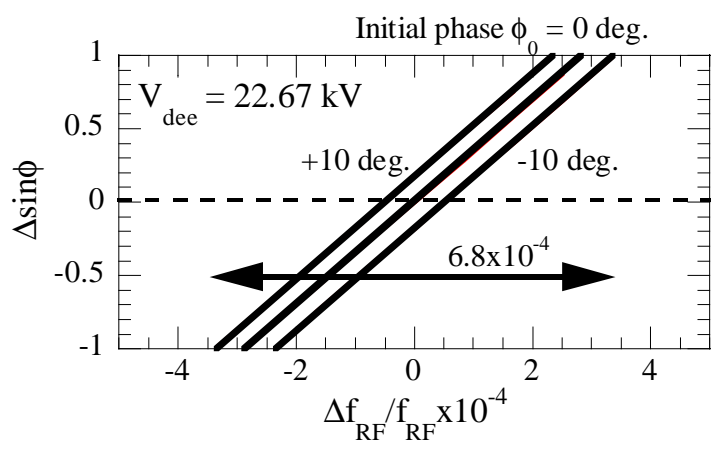

Figure 1: Dependence of the change in phase at the extraction radius on the frequency shift.

$$
\Delta \sin \phi \approx 2 \pi h \frac{\Delta f_{R F}}{f_{R F}} \frac{r^{2}}{2} \frac{m c^{2}}{\Delta E}\left(\frac{2 \pi f_{R F}}{h c}\right)^{2},
$$

where $\mathrm{r}$ is a radius, $\Delta \mathrm{E}$ the peak energy gain. Relation between the frequency shift and the change in phase at the extraction radius is shown in Fig.1. The $\Delta \sin \phi$ is approximately proportional to the frequency change. The frequency range for the beam with an initial phase width of 20 degrees RF is $6.8 \times 10^{-4}$. Assuming that a half width of the frequency range gives FWHM, the M/Q resolution is estimated at $\mathrm{R}=2900$. The resolution for the extracted beam will be higher than this, since the beam is defined by acceptance of the extraction system [4].

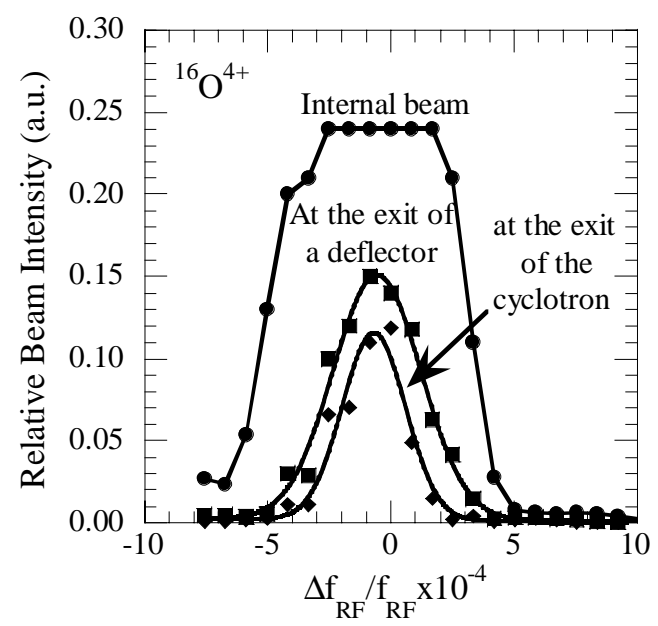

Figure 2: Dependence of the beam intensity on the frequency shift. The frequency shift range is estimated at $8.4 \times 10^{-4} \mathrm{FWHM}$ for the internal beam, $4.2 \times 10^{-4} \mathrm{FWHM}$ for the beam at the exit of the deflector and $3.0 \times 10^{-4}$ FWHM for the beam extracted from the cyclotron.
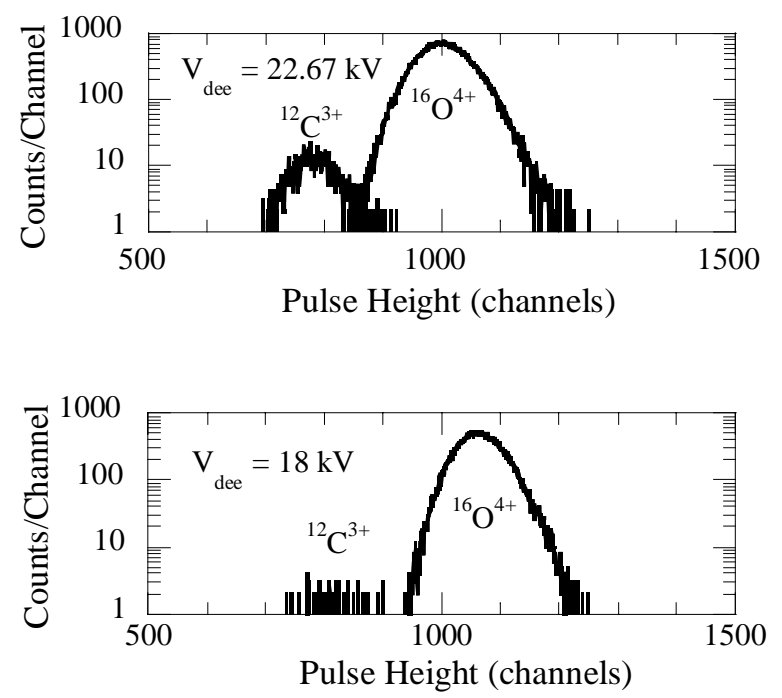

Figure 3: Pulse height spectra of the ${ }^{12} \mathrm{C}^{3+}$ and ${ }^{16} \mathrm{O}^{4+}$ cocktail beam obtained at dee voltages of 22.67 $\mathrm{kV}$ (upper) and $18 \mathrm{kV}$ (lower). The yield ratio of the ${ }^{12} \mathrm{C}^{3+}$ ions to the ${ }^{16} \mathrm{O}^{4+}$ ions is $1.6 \times 10^{-2}$ at $22.67 \mathrm{kV}$ and $0.29 \times 10^{-2}$ at $18 \mathrm{kV}$.

\subsection{Experimental Estimation}

Variation of beam intensity depending on the frequency shift is shown in Fig. 2. The width of the frequency shift for the internal beam just before extraction was $8.4 \times 10^{-4}$ FWHM. In this measurement two sets of phase defining slits were placed at the position mostly evacuating from a beam. The phase width of the internal beam was larger than 20 degrees RF. The frequency range became a half at the exit of a deflector, and was further reduced to $3.0 \times$ $10^{-4} \mathrm{FWHM}$ after extracting from the cyclotron due to the acceptance of the extraction system. The resolution estimated from the frequency range is around 3300 that is insufficient for separating the ${ }^{12} \mathrm{C}^{3+}$ and ${ }^{20} \mathrm{Ne}^{5+}$ ions from the ${ }^{16} \mathrm{O}^{4+}$ beam.

\subsection{Improvement of the Resolution}

The amount of the change in phase is inversely proportional to the energy gain as given in Eq. (3). In order to separate the particles with different M/Q values before extraction, decrease in energy gain, that is increase in turn numbers, is required. Pulse height spectra of the ${ }^{12} \mathrm{C}^{3+}$ and ${ }^{16} \mathrm{O}^{4+}$ cocktail beam obtained by a plastic scintillator at a dee voltage of $22.67 \mathrm{kV}$ and 18 $\mathrm{kV}$ are shown in Fig. 3. The yield ratio of the ${ }^{12} \mathrm{C}^{3+}$ to ${ }^{16} \mathrm{O}^{4+}$ went down to one fifth by simply changing the dee voltage. Careful optimization of parameters is required for further improvement of the resolution. 


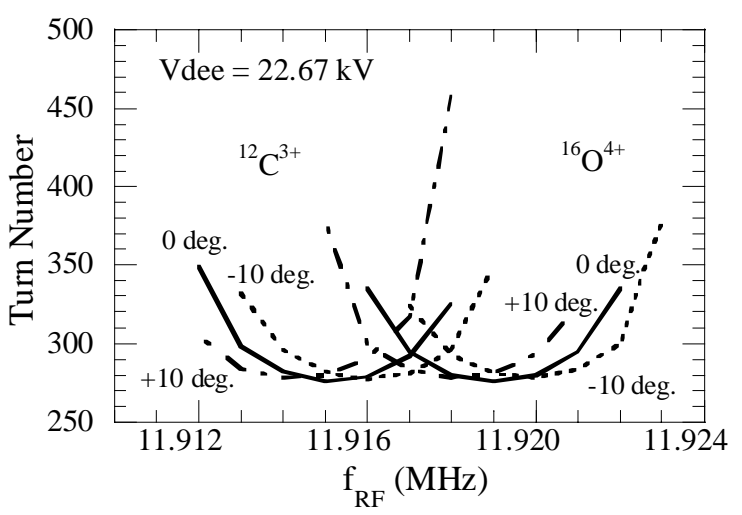

Figure 4: Increase in turn numbers for the ${ }^{12} \mathrm{C}^{3+}$ and ${ }^{16} \mathrm{O}^{4+}$ beams with an initial phase width of 20 degrees caused by the frequency shift.

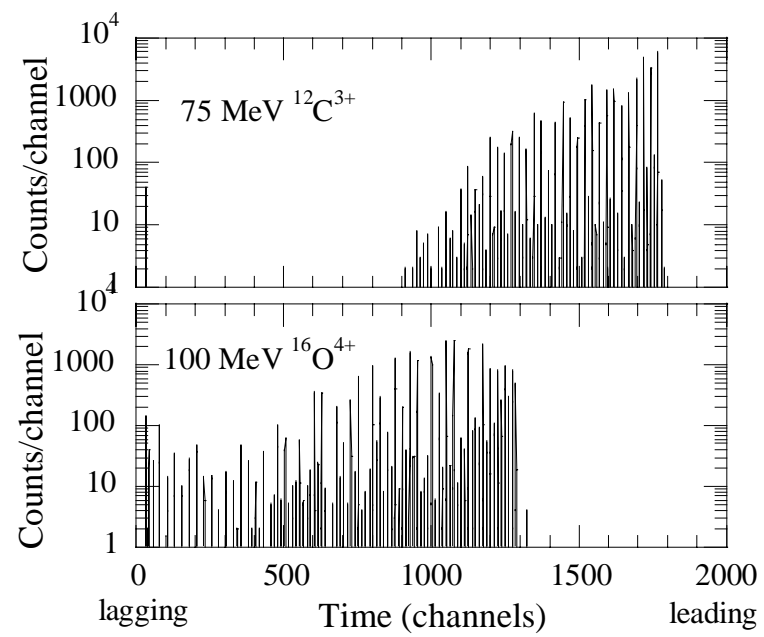

Figure 5: Beam pulses of the ${ }^{12} \mathrm{C}^{3+}$ (upper) and ${ }^{16} \mathrm{O}^{4+}$ (lower) ions at the optimum frequency of 19.916 $\mathrm{MHz}$ measured with a plastic scintillator.

\section{TURN NUMBERS}

The amount of change in phase for particles with different $\mathrm{M} / \mathrm{Q}$ values is linearly increasing with turn numbers as given in the Eq. (2). The calculated turn numbers of the particles reaching to the extraction radius are shown in Fig. 4. The minimum turn number is obtained at the optimum frequency for each ion species.

An increment in turn numbers for the frequency shift of $2.5 \times 10^{-4}\left(\Delta \mathrm{f}_{\mathrm{RF}}=3 \mathrm{kHz}\right)$ is around 60 . The energy gain of the particles is gradually decreasing due to the drift of the beam phase caused by the frequency shift. The particles having lower energy stay in orbits at smaller radii, which causes radial spread of a beam bunch. The radial spread at the entrance of the deflector is larger than the aperture of the deflector. Thus the beam bunch is chopped by the deflector and extracted with multiple turns. Time spectra of the extracted beam at the optimum frequency for ${ }^{12} \mathrm{C}^{3+}$ are shown in Fig. 5. The beam was pulsed before injection using a trapezoidal voltage chopper (P chopper) [5] so that the multiple turns could be observed. The ${ }^{12} \mathrm{C}^{3+}$ ions were extracted first, and a pulse train of the ${ }^{16} \mathrm{O}^{4+}$ ions appeared 41 turns later. The delay of the beam pulses was consistent with the calculation result shown in Fig. 4.

\section{ELIMINATION OF IMPURITY IONS IN PRACTICAL OPERATION}

In order to avoid intermixture of the different ion species in the extracted beam, a grouping of cocktail ions with sufficiently different $\mathrm{M} / \mathrm{Q}$ values is required for the cocktail acceleration. The choice of the ion species for such grouping is, however, limited, which is lacking in variety for the use of multiple ion species.

Improvement of the M/Q resolution mentioned in the section 2.3 is an orthodox way for reducing the impurity ions. But additional time for parameter optimization might be necessary. This is contrary to the fast change of the beam.

For the practical operation, simplification of the ion species injected into the cyclotron is the easiest way for purification of the beam. In order to save the time for changing a gas supplied to the ECR ion source, the gas supplying system was modified. The capacity of gas pipes was minimized, and a nitrogen gas having an M/Q value far from 4 was used as a common support gas to save the time for purging in the pipes. As a result, the ion species can be changed within ten minutes.

The ${ }^{84} \mathrm{Kr}^{21+}$ beam contained some metallic ions coming from a plasma chamber of the ECR ion source, since the intensity of the ${ }^{84} \mathrm{Kr}^{21+}$ ions is low for our ECR ion source. Fortunately the krypton gas consists of some stable isotopes, and the M/Q values of the isotope ions is sufficiently different from the impurity ions. Optimizing the frequency to the isotope ions can purify the krypton beam.

\section{REFERENCES}

[1] K. Arakawa, et al., Proc. $13^{\text {th }}$ Int. Conf. On Cyclotron and Their Application, Vancouver, Canada, pp.119-122 (1992)

[2] M.A. McMahan, et al., Nucl. Instr. and Meth. A253 (1986)1

[3] M. Fukuda, et al., Proc. $11^{\text {th }}$ Symposium on Accelerator Science and Technology, Harima Science Garden City, Japan, pp.139-141 (1997)

[4] G. Ryckewaert, et al., Proc. $13^{\text {th }}$ Int. Conf. On Cyclotron and Their Application, Vancouver, Canada, pp.737-740 (1992)

[5] W. Yokota, et al., Rev. Sci. Instrum. 68 (1997) 1714 